\title{
Differential Rotation and Dynamics of the Solar Interior
}

M.J. Thompson, J. Toomre, E.R. Anderson, H.M. Antia, G. Berthomieu, D. Burtonclay, S.M. Chitre, J. Christensen-Dalsgaard, T. Corbard, M. DeRosa, C.R. Genovese, D.O. Gough, D.A. Haber, J.W. Harvey, F. Hill, R. Howe, S.G. Korzennik, A.G. Kosovichev, J.W. Leibacher, F.P. Pijpers, J. Provost, E.J. Rhodes, Jr., J. Schou, T. Sekii, P.B. Stark, P.R. Wilson

Splitting of the Sun's global oscillation frequencies by large-scale flows can be used to investigate how rotation varies with radius and latitude within the solar interior. The nearly uninterrupted observations by GONG yield oscillation power spectra with high duty cycle and signal-to-noise ratio. Frequency splittings derived from GONG observations confirm that the variation of rotation rate with latitude seen at the surface carries through much of the convection zone, at the base of which is an adjustment layer leading to latitudinally independent rotation at greater depths. A distinctive shearing layer just below the surface is discernible at low to mid latitudes.

M.J. Thompson, R. Howe, Astronomy Unit, Queen Mary \& Westfield College, University of London. J. Toomre, M. DeRosa, D.A. Haber, JILA, University of Colorado. E.R. Anderson, J.W. Harvey, F. Hill, J.W. Leibacher, National Solar Observatory, Tucson. H.M. Antia, S.M. Chitre, Tata Institute of Fundamental Research, Bombay. G. Berthomieu, T. Corbard, J. Provost, Observatoire de la Cote d'Azur, Nice. D. Burtonclay, P.R. Wilson, School of Mathematics, University of Sydney. J. Christensen-Dalsgaard, F.P. Pijpers, Theoretical Astrophysics Center, Aarhus University. C.R. Genovese, Department of Statistics, Carnegie Mellon University. D.O. Gough, T. Sekii, Institute of Astronomy, University of Cambridge. S.G. Korzennik, Harvard-Smithsonian Center for Astrophysics. A.G. Kosovichev, J. Schou, CSSA, Stanford University. E.J. Rhodes, Jr., Department of Physics \& Astronomy, University of Southern California. P.B. Stark, Department of Statistics, University of California, Berkeley. 
On the timescale of stellar evolution, the Sun is a middle-aged star. The surface rotation rates of young solar-type stars are observed to be up to 50 times that of the Sun. It is therefore believed that the Sun has been losing angular momentum over its lifetime through its magnetized wind, thereby gradually spinning down its outer convection zone and probably the bulk of its interior. The effectiveness of the overall spindown of the star is difficult to estimate from stellar evolution theory, for one must cope with delicately balanced circulations and instabilities that would tend to mix the interior, and magnetic fields that may retard or modify such processes (1). This has led to suggestions that the Sun might still possess a rapidly rotating core, perhaps highly magnetized, reflecting its primordial past. The apparent deficit of neutrinos coming from the Sun's energy-generating core has also prompted ideas for readjusting the chemical composition and stratification in models of the nuclear-burning core, and these have implications for the mixing of angular momentum there (2).

Tracking of surface features has shown that the Sun does not rotate as a solid body: it rotates once in about 25 days near the equator and in about 33 days near the poles. Further, the rotation rate of sunspots at mid-latitudes is somewhat faster than that deduced from Doppler shifts of the surface plasma, suggesting that the magnetic fields of the spots may be rooted to more rapidly rotating plasma deeper down in the convection zone, which occupies the Sun's outer $30 \%$ by radius (3). Theoretical studies indicate that turbulent compressible convection couples with rotation to redistribute the angular momentum away from simple solid-body rotation, leading to differential rotation and meridional circulations. Moreover, the interplay of turbulent motions and rotation with magnetic fields is generally believed to be responsible for magnetic dynamo action that leads to the observed 22-year cycles of sunspots and solar magnetic activity (4). During the past decade, helioseismology has begun to provide the means to estimate the rotation profile of the interior of the Sun. The helioseismic findings are not compatible with the predictions of most theoretical models of the rotation profile set up by turbulent convection in the Sun's envelope (5), which raises serious doubts about our current understanding of global-scale solar convection. Here, we use the nearly continuous observations by GONG to probe the dynamical state of the solar convection zone and the deeper radiative interior. 
The horizontal structure of each global mode of oscillation of the Sun is described by a spherical harmonic $Y_{l}^{m}$ of degree $l$ and order $m$, and the vertical structure by $l$ and the radial order $n$. Opposite signs of $m$ correspond to modes propagating in opposite directions around the Sun's equator. In a spherically symmetric star the frequencies depend upon $n$ and $l$ but not on $m$, so for each $(n, l)$ pair there is a $(2 l+1)$-fold degeneracy. Rotation breaks the spherical symmetry and lifts the degeneracy. Advection causes a wave propagating with the Sun's rotation to have a higher measured frequency than a similar wave propagating against the rotation. The difference in frequency of a pair of oppositely propagating modes is proportional to $m$ times a weighted average of the rotation rate $\Omega(r, \theta)$ in the region of radius $r$ and latitude $\theta$ where the modes have appreciable amplitude. We define the frequency splitting $\Delta \nu_{n l m}$ to be half the value of this difference. Each frequency splitting measures a longitudinal and temporal average of the zonal flows over the period of the observations (6). Different modes have different spatial sensitivity, so the observed frequency splittings can be used to make inferences about spatial variations in $\Omega$.

There were several early attempts (7) to measure rotational splittings, but the first definitive results yielding estimates of $\Omega$ over a range of depths were obtained from observation of intermediate-degree sectoral $(m= \pm l)$ modes (8). The frequency splittings yielded inferences for $\Omega$ close to the equatorial plane, suggesting that much of the interior of the Sun rotates slightly less rapidly than the surface, while the central region appears to rotate more rapidly. Such data also indicated that the quadrupole moment $J_{2}$ of the Sun's exterior gravitational field is consistent with General Relativity. These studies were soon followed by full-disk imaging observations that yielded information on a wide variety of tesseral $(0<|m|<l)$ modes, thereby providing estimates of the internal rotation away from the equatorial plane $(9-14)$.

Helioseismic data have revealed that the surface rotation rate persists through much of the convection zone on radial lines, and that there is a transition at or near the base of the convection zone to rotation that is independent of latitude. The sidereal rotation rate beneath the convection zone - roughly $440 \mathrm{nHz}$ - is such that the latitudinally averaged specific angular momentum appears to be nearly constant across the convection zone 
boundary, suggesting that there is no net torque exerted across the transition (15). The rotation in the radiative interior beneath the convective envelope seems to be consistent with rigid-body rotation. The rotation rate of the core is less certain: some reported low-degree splittings favor a rotation rate faster than the equatorial rate at the surface (16), and others a somewhat slower rate (17).

The convection in early numerical simulations of rotating convection in spherical shells was dominated by columnar roll-like cells orientated in the north-south direction. The tilting of these cells yielded Reynolds stress terms that drove zonal flows, which appeared as differential rotation (18). The convection models predicted that $\Omega$ was nearly constant along the axes of the columnar cells, and thus that angular velocity was nearly constant on cylinders aligned with the rotation axis and decreased with depth in the equatorial plane. In contrast, the helioseismic data imply that angular velocity is roughly constant on radial lines. Recent numerical studies capable of describing more turbulent compressible flows have yielded more intricate profiles for $\Omega$, although there is still some tendency for angular velocity to be constant on cylinders close to the equator (19). A likely explanation of the discrepancy between simulation and helioseismic inferences is that the spatial resolution in the theoretical convection models is capable of describing only mildly turbulent flows, whereas fully developed turbulence involving coherent structures embedded in otherwise chaotic flow fields may yield quite different mean flows and rotation profiles.

\section{Interpreting Frequency Splittings}

The splitting $\Delta \nu_{n l m}$ caused by the rotation $\Omega(r, \theta)$ can be written as

$$
\Delta \nu_{n l m}=\frac{m}{2 \pi} \int K_{n l m}(r, \theta) \Omega(r, \theta) r d r d \theta
$$

where $K_{n l m}$ are weighting functions, known as rotation kernels (Fig. 1). The kernels reflect the vertical and horizontal structure of the mode eigenfunctions. Because the spherical harmonics are either purely symmetric or purely antisymmetric about the equatorial plane, the amplitude of oscillation is symmetric about the equator and hence so too are the rotation kernels. Consequently, Eq. 1 implies that the splitting is sensitive only to that component of 
$\Omega$ that is symmetric about the Sun's equatorial plane. Other contributions to the splitting might come from latitudinal variations in structure or a magnetic field that is axisymmetric about the rotation axis. Such contributions are distinguishable from the rotation splitting because only the latter is antisymmetric in $m$.

Because the integrals of the rotation kernels are close to unity, the overall magnitude of the observed splittings readily provides an estimate of the Sun's internal rotation rate. A simple way to proceed further would be to model the internal rotation profile with a few parameters, use Eq. 1 to compute the splittings corresponding to such a profile, and adjust the parameters to match the observed splittings. A more systematic approach would be to note that different modes are sensitive to the rotation in different parts of the interior in a way that varies systematically with mode parameters. Modes sample the rotation rate in a cavity that extends from the surface down to a depth that increases with $\nu / L$, where $\nu$ is the frequency of the mode and $L=\sqrt{l(l+1)}$. Hence low-degree modes are sensitive to rotation from the surface to the core, whereas high-degree modes are only sensitive to the rotation close to the surface. In the latitudinal direction, modes sample the rotation between latitudes $\pm \cos ^{-1}(m / L)$. Thus the dependence of the splittings on $\nu / L$ and $m / L$ may guide the construction of forward models for the rotation as a function of radius and latitude. For instance, splittings of modes with $m \approx \pm l$ show how the near-equatorial rotation varies with depth (Fig. 2). Each value of $\nu / L$ corresponds to a radius $r_{\mathrm{t}}$ to which such a mode penetrates, and thus the splitting represents a weighted average of the equatorial rotation between the surface and the radial location $r_{\mathrm{t}}$. Thus the GONG data reveal that in the equatorial regions the rotation rate first increases with depth and then decreases. Suitable combinations of splittings sample the rotation rate at other latitudes (20). A data combination appropriate for a latitude of $30^{\circ}$ (Fig. 2) shows that the Sun rotates less rapidly at this latitude than at the equator, as is also observed from surface measurements. The rotation initially increases with depth at this latitude, but less strongly than at the equator.

Alternatively, one can use one of several inverse techniques. Here we apply two such techniques to the GONG data. Suppose that one can find a linear combination of the rotation kernels that is peaked at some chosen location within the Sun and small elsewhere 
(Fig. 1). Since Eq. 1 is linear, the same linear combination of the measured splittings provides a weighted average of the internal rotation rate in the Sun, weighted by the averaging kernel. Provided the averaging kernel is localized, it yields an estimate of the rotation rate in some localized region of the solar interior. By repeating this for different target locations, one can build a picture of the rotation rate inside the Sun. The construction of localized kernels is the essence of the Optimally Localized Averages (OLA) method, also known as the Backus-Gilbert method (22,23). Another approach to inversion is to fit a parametric model of the internal rotation rate to the data with a least squares fit. Because our problem is ill-conditioned, we use a regularized least squares (RLS) method, with a penalty function that penalizes solutions that vary on small length scales. In our formulation, the solution at each point is a linear combination of the data, and thus provides an estimate of the rotation rate as sampled by the corresponding linear combination of the rotation kernels, just as in OLA (24).

\section{Inferences From GONG Data}

Inversions of GONG splitting data by OLA and RLS methods (Fig. 3), in the region where they can reasonably be determined with the four-month data set, are in good agreement $(25-27)$. In the convection zone above latitude $\sim 30^{\circ}$, the data show that the rotation rate at fixed latitude is roughly independent of depth, so that the variation with latitude is similar to that seen at the surface. Near the equator, the rotation rate increases just below the surface and reaches a maximum at roughly $r=0.95 R$ (where $R$ is the radius of the Sun). It then gradually decreases with depth in the convective envelope. At the base of the convection zone near $r=0.7 R$, there is a pronounced transition at all latitudes to nearly uniform rotation at greater depths (Fig. 4). The structure of the transition is not resolved by the data. Thus the GONG data support earlier deductions that the surface-like differential rotation is smoothed out near the base of the convection zone and the rotation below appears to become independent of latitude. The data from GONG currently permit reliable inferences only to a depth of about $r=0.4 R$, and the use of global modes yields little information near the poles (28). 
These inversions indicate the presence of two layers with strong horizontal shear in the mean azimuthal velocity, one positioned just below the surface and at the other at the base of the convection zone. In contrast, gradients in $\Omega$ are mild in the rest of the convective envelope. These results are in accord with analyses of earlier helioseismic data, although our confidence in the inferences made from the nearly continuous GONG data is enhanced by the improved determination of frequency splittings with $m$, because GONG spectra do not suffer from daily sidelobes, which plague single-site, ground-based observations.

The presence of an upper shear layer is consistent with the observation that at low latitudes sunspots and supergranulation patterns rotate faster than the surface gas (29). Perhaps near-surface convective motions with overturning times short compared to the rotation period (and thus only mildly influenced by Coriolis forces) conserve their specific angular momentum as they move radially inward and toward the rotation axis, leading to a faster latitudinally varying rotation rate than at the solar surface (30). Alternatively, recent numerical simulations of highly turbulent rotating convection within limited domains (31) exhibit prominent shear layers close to where the radial velocity vanishes on surfaces that bound the zone of convection, and across which no net stresses are communicated. The convection models yield such shear layers only in the presence of intense small-scale turbulence; and such levels of turbulence have not yet been attained in models of convection in full spherical shells spanning a broader physical domain. Yet the real solar convection zone must be far more turbulent than any flows studied by simulation (19). Moreover, it possesses the added complexities of magnetic fields and the ionization zones of hydrogen and helium close to its surface. It has been conjectured that the combined presence of the ionization zones and the rapid variation in stratification near the surface may contribute to the lateral deflection of large-scale convective motions, leading possibly to substantial horizontal flows below the surface and only relatively feeble motions in the directly observable atmosphere. Such giant cells are expected to possess horizontal dimensions comparable to the depth of the convecting shell. Giant cells have not yet been observed with any certainty, but the structure of the upper shearing layer detectable from helioseismic data, and the manner in which it varies with latitude, may eventually provide clues to the nature of coherent long-lived structured flows that may coexist with small-scale turbulence within the 
solar convection zone. The extent to which the shear layer extends to higher latitudes is uncertain from current observations using the global modes: there is a hint in the inversions that the shear changes sign at mid-latitudes (Fig. 3).

An alternative way of probing the upper shear layer is provided by helioseismic analyses of acoustic wave fields within a localized area, using time-distance or ring-diagram methods (32). Ring analysis applied to some GONG data is shown in Fig. 5. Mosaics of such measurements may be used to map out the mean flows with depth beneath each patch, using a one-dimensional inversion in depth. The local mean horizontal flows appear to spiral with depth in the immediate sub-surface layers, suggesting that the shearing flows may possess more intricate structure than that deduced from the properties of the global modes. Such local-area techniques would benefit from higher spatial resolution that future upgrades to GONG may provide.

The second shear layer at the base of the convection zone (Figs. 3, 4) is a transition from the angular velocity profile of the convective envelope to a profile of possible solidbody rotation in the deeper radiative interior (33). This transition layer is likely to be dynamically complicated, for it is just below, or may even be part of, the overshooting region in which penetrating convective motions, probably in the form of discrete plumes, are rapidly decelerated as they encounter the stable stratification of the interior. Helioseismic findings that the radial gradient of the rotation rate is small in the convection zone itself has shifted attention to this transition layer as the site of the magnetic dynamo (34). One would like to know the nature of the stratification and the mean shearing flows achieved in the layer in order to assess how thick a region is available for making and storing the magnetic fields. However, inferences about the transition in rotation pattern and in mean stratification are still uncertain because the resolution permitted by the helioseismic data is still too poor there. With available data, the characteristic full width at half maximum (FWHM) of an averaging kernel peaked at low latitudes near the base of the convection zone is roughly $0.1 R$. This is essentially the radial resolution, and it is not possible to say whether the transition is abrupt or broad (35). 
Beneath this transition layer, down to radius $0.4 R$ where we can reliably make inferences with this data set, the rotation appears to be consistent with solid-body rotation. The latter may seem plausible since it represents the lowest energy state for a specified total angular momentum. Yet how that may be achieved within the Sun is uncertain, for models incorporating angular momentum transport by circulations and hydrodynamic instabilities favor an interior, and especially a core, rotating considerably faster than the solar surface (1). A weak connected magnetic field pervading the whole of the radiative interior could cause this region to rotate uniformly (36): one would presume that the field does not penetrate the shear layer, where it would be stretched, but has been expelled by the turbulence (37). It has also been suggested that internal gravity waves excited by penetrative convection propagate inward, transporting angular momentum radially (38). However, the observed limited depletion of lithium in the solar envelope places important constraints on such spin-down processes as would also mix material from the convection zone to the hotter regions beneath, where the lithium would be destroyed (39). Helioseismic probing of the deep radiative interior should help to resolve these questions, for we can expect to improve greatly the resolution and the range of depths over which we can make reliable inferences as we accumulate more data from GONG.

Similar results to those in Figs. 3,4 have also been derived from the GONG data set using 1.5D inversion methods and by forward modeling. These strengthen our conclusions regarding the rotation rate. It must be borne in mind that the rotation rate we determine using the splittings of the global modes is an average in longitude and time, and is only a north-south symmetric average. Furthermore, the limited spatial resolution (40) results in smoothing of the inferred rotation rate of the Sun. The examples of Jupiter and Saturn, which are likewise rotating, convecting bodies, suggest that the Sun might possess strong zonal jets and structured flows, in addition to broader variations of differential rotation. Observing for longer periods will enable frequencies to be measured even more accurately, with consequent improved spatial resolution (41). However, the mean flows within the Sun might not be steady over periods of a year or more, and inverting time-averaged splitting data will then only provide a picture of the time-averaged dynamics. The analyses of wave 
properties over more localized areas using time-distance or ring-diagram approaches (32), both of which can be accomplished over shorter intervals in observing time, may serve as an important complement to the sampling afforded by the use of the global modes. The dynamics of the solar convection zone and deeper interior is likely to involve a diverse range of temporal and spatial scales of behavior (19), the resolution of which will require the extended imaging data sets that are now becoming available from GONG and associated helioseismic experiments. 


\section{References and Notes}

1. Rotational histories of low-mass stars like the Sun are discussed in L.W. Hartmann and R.W. Noyes, Annu. Rev. Astron. Astrophys. 25, 271 (1987); M.H. Pinsonneault, S.D. Kawaler, S. Sofia, P. Demarque, Astrophys. J. 338424 (1989); B. Chaboyer, P. Demarque, M.H. Pinsonneault, ibid. 441, 865 (1995).

2. Possible dynamical processes in solar core are discussed in D.O. Gough, in SolarTerrestrial Relationships and the Earth Environment in the Last Millenium, G. Castagnoli-Cini, Ed. (Soc. Italiana di Fisica, Bologna, 1988), pp. 90-132; W.J. Merryfield, J. Toomre, D.O. Gough, Astrophys. J. 367, 658 (1991); S. Ghosal and E.A. Spiegel, Geophys. Astrophys. Fluid Dynam. 61, 161 (1991). Computation of modifications to the neutrino flux are considered in D.O. Gough, Ann. New York Acad. Sci. 647, 119 (1991).

3. The average sidereal rotation rate $\Omega / 2 \pi$ from photospheric Doppler measurements is about $452 \mathrm{nHz}$ at the equator, $432 \mathrm{nHz}$ at $30^{\circ}$, and $369 \mathrm{nHz}$ at $60^{\circ}$ [Ulrich et al., Solar Phys. 117, 291 (1988)]. The rotation rate determined by tracking sunspots varies with the size of the spot group [R. Howard, P. Gilman, P. Gilman, Astrophys. J. 283, $373(1984)$ ] or with the age of the spots [E. Nesme-Ribes et. al., Astron. Astrophys. 274, 563 (1993)]. Average sunspot rotation rates are quoted as $467 \mathrm{nHz}$ and $465 \mathrm{nHz}$ respectively near the equator, and $444 \mathrm{nHz}$ and $450 \mathrm{nHz}$ at $30^{\circ}$. See also E.H. Schröter, Solar Phys. 100, 141 (1985).

4. P.A. Gilman in Physics of the Sun, 1, P.A. Sturrock, T.E. Holzer, D.M. Mihalas, R.K. Ulrich, Eds. (Reidel, Dordrecht, Netherlands, 1986), pp. 95-160; S.K. Solanki, Space Sci. Rev. 63, 1 (1993); F. Cattaneo, in Solar Magnetic Fields, M. Schüssler and W. Schmidt, Eds. (Cambridge Univ. Press, Cambridge, 1993), pp. 261-275; P.R. Wilson, Solar and Stellar Activity Cycles (Cambridge Univ. Press, Cambridge, 1994).

5. D.O. Gough and J. Toomre, Annu. Rev. Astron. Astrophys. 29, 627 (1991). 
6. The effect of large-scale flows on mode frequencies and linewidths is discussed by E.M. Lavely and M.H. Ritzwoller, Astrophys. J. 403, 810 (1993).

7. Low-degree oscillations were studied through Doppler shifts in light from the entire solar disk by A. Claverie et. al, Nature 293, 443 (1981); G. Grec, E. Fossat, M.A. Pomerantz, Solar Phys. 82, 55 (1983); and through fluctuations in the limb-darkening function by H. Hill, R.J. Bos, P.R. Goode, Phys. Rev. Lett. 49, 1794 (1982).

8. Sectoral modes were studied with a cylindrical lens folding the solar image into the entrance slit of a spectrograph aligned with the solar equator [T.L. Duvall, Jr. and J.W. Harvey, Nature 310, 19 (1984)], and splitting data inverted by T.L. Duvall, Jr. et al., ibid. 310, 22 (1984).

9. Frequency splittings for intermediate degree modes using the Fourier Tachometer, the progenitor of the GONG instrument, were reported by T.M. Brown and C.A. Morrow, Astrophys. J. 314, L21 (1987), and inverted by T.M. Brown et al., Astrophys. J. 343, 526 (1989); see also T.M. Brown, Nature 317, 591 (1985).

10. Intermediate-degree frequencies and splittings from Big Bear Solar Observatory, using Doppler imaging with a tuneable birefringent filter, were reported for a 1986 campaign in K.G. Libbrecht, Astrophys. J. 336, 1092 (1989), with further campaigns in 1988-90 [K.G. Libbrecht and M.F. Woodard, Nature 345, 779; M.F. Woodard and K.G. Libbrecht, Astrophys. J. 402, L77]. Rotation inversions were discussed in J. Christensen-Dalsgaard and J. Schou, in Seismology of the Sun and Sun-like Stars, E.J. Rolfe, Ed. (ESA SP-286, ESA, Noordwijk, Netherlands, 1988), pp. 149-153; W.A. Dziembowski, P.R. Goode, K.G. Libbrecht, Astrophys. J. 337, L53 (1989); D.O. Gough et al., Astron. Soc. Pac. Conf. Ser. 40, 93 (1993); M.F. Woodard and K.G. Libbrecht, Science 260, 1778 (1993).

11. Intermediate and high-degree mode parameters deduced from South Pole Ca II intensity observations were reported in T.L. Duvall, Jr., J.W. Harvey, M.A. Pomerantz, Nature 321, 500 (1986); —-, et al. Astrophys. J. 324, 1158 (1988); - , J.W. Harvey, S.M. Jeffries, M.A. Pomerantz, ibid. 373, 308 (1991); S.M. Jeffries et al., ibid. 
377, 330 (1991); T.L. Duvall, Jr. et al., ibid. 410, 829 (1993). That instrumentation now serves as the High Degree Helioseismometer (HDH) [K.T. Bachmann, T.L. Duvall, Jr., J.W. Harvey, F. Hill, ibid. 443, 837 (1995)].

12. Extensive sets of intermediate and high-degree frequencies and splittings observed from Mt. Wilson with a Doppler magneto-optical analyzer have been reported by S. Tomczyk et al., in Seismology of the Sun and Sun-like Stars, E.J. Rolfe, Ed. (ESA SP-286, ESA, Noordwijk, Netherlands, 1988), pp. 141-147, and inverted by S.G. Korzennik et al. ibid., pp. 117-124; E.J. Rhodes, Jr. et al., Astrophys. J. 351, 687 (1990). For inferences from 1988 and 1990 campaigns, see S.G. Korzennik et al., in Proc. Oji Intern. Seminar Prog. of Seismology of the Sun and Stars, Y. Osaki and H. Shibahashi, Eds. (Springer Verlag, Berlin, 1990), pp. 341-348; P.R. Goode et al., Astrophys. J. 367, 649 (1991); S.G. Korzennik, A. Cacciani, E..J. Rhodes, Jr., Astron. Soc. Pac. Conf. Ser. 42, 201 (1993); S.G. Korzennik et al., ibid. 76, 12 (1995).

13. The LOWL magneto-optical analyzer [S. Tomczyk et al., Solar Phys. 159, 1 (1995)] has produced a long campaign of observations from Mauno Loa in Hawaii. Rotation inversions were presented by S. Tomczyk, J. Schou, M.J. Thompson, Astrophys. J. 448, L57 (1995).

14. Recent interpretation of low- $l$ splittings from the IRIS and BiSON networks and from IPHIR have been reported by E. Fossat et al., Astron. Soc. Pac. Conf. Ser. 76, 24 (1995); Y. Elsworth et al., ibid., 76, 43 (1995), —-, Nature 376, 669 (1995); and T. Toutain, ibid., 76, 34 (1995), respectively.

15. T.M. Brown et al., Astrophys. J. 343, 526 (1989); W.A. Dziembowski and P.R. Goode, Astron. Soc. Pac. Conf. Ser. 42, 225 (1993).

16. A. Claverie et al., Nature 293, 443 (1981); A. Jiménez et al., Astron. Astrophys. 435,874 (1994).

17. T. Toutain and A.G. Kosovichev, Astron. Astrophys. 284, 265 (1994); T. Appourchaux et al., in Fourth SOHO Workshop Helioseismology, T. Hoeksema, V. Domingo, 
B. Fleck, B. Battrick, Eds. (ESA SP-376, Vol. 2, ESA, Noordwijk, Netherlands, 1995), pp. 265-270; Y. Elsworth et al., Nature 376, 669 (1995); S. Tomczyk, J. Schou, M.J. Thompson, Bull. Astron. Soc. India, in press (1996).

18. Numerical simulations of convection constrained by rotation within spherical shells were reported by G.A. Glatzmaier, Astrophys. J. 291, 300 (1985); P.A. Gilman and J. Miller, Astrophys. J. 61, 585 (1986); G.A. Glatzmaier, in The Internal Solar Angular Velocity, B.R. Durney and S. Sofia, Eds. (Reidel, Dordrecht, Netherlands, 1987), pp. 263-274. Modified mixing-length and anisotropic diffusion models can also be used to estimate Reynolds stresses of rotating convection, as in G. Rüdiger, Geophys. Astrophys. Fluid Dynam. 16, 239 (1980); G. Belvedere, L. Paterno, M. Stix, Astron. Astrophys. 86, 40 (1980); D.H. Hathaway, Astrophys. J. 276, 316 (1984); B.R. Durney, ibid. 378, 378 (1991).

19. N. Brummell, F. Cattaneo, J. Toomre, Science 269, 1370 (1995); G.A. Glatzmaier and J. Toomre, Astron. Soc. Pacific Conf. Ser. 76, 200 (1995).

20. The $m$-dependence of the splittings for given $n$ and $l$ can be approximately parametrized by a low-order polynomial in $m / L: \Delta \nu_{n l m}=\sum_{k=1}^{N} a_{k}(n, l) \mathcal{P}_{k}^{(l)}(m)$. The $\mathcal{P}_{k}^{(l)}(m)$ are polynomials in $m$ of degree $k$ : a common choice is $\mathcal{P}_{k}^{(l)}(m)=$ $L P_{k}(m / L)$, where $P_{k}$ is a Legendre polynomial. The coefficients $a_{k}$ do then depend upon the number $N$ of terms included in the expansion. Here we use orthogonal polynomials as defined in (21) and in M.H. Ritzwoller and E.M. Lavely, Astrophys. J. 369, 557 (1991), which are asymptotically equal to the previous choice of polynomials and yet make the coefficients almost independent of $N$. The traces in Fig. 2 show $a_{1}+a_{3}+a_{5}$ (equatorial) and $a_{1}-\frac{1}{4} a_{3}-\frac{19}{16} a_{5}$ (latitude $30^{\circ}$ ). Further details of the combining of data are given in T.M. Brown et al., Astrophys. J., 343, 526 (1989); P.R. Wilson and D. Burtonclay, Astrophys. J. 438, 445 (1995).

21. J. Schou, J. Christensen-Dalsgaard, M.J. Thompson, Astrophys. J. 433, 389 (1994).

22. The combining of kernels as in Optimally Localized Averages was introduced by G. Backus and F. Gilbert, Geophys. J. R. Astron. Soc. 16, 169 (1968); G. Backus 
and F. Gilbert, Phil. Trans. R. Soc. London, Ser. A 266, 123 (1970). Its use in helioseismology is discussed in D.O. Gough, Solar Phys. 100, 65 (1985); and the particular formulation known as SOLA in F.P. Pijpers and M.J. Thompson, Astron. Astrophys. 262, L33 (1992).

23. OLA is computationally expensive, requiring the inversion of a matrix of order the number of data. The $1 \otimes 1$ inversion approach [T. Sekii, Mon. Not. R. Astron. Soc. 264, 1018 (1993); T. Sekii, in Fourth SOHO Workshop Helioseismology, T. Hoeksema, V. Domingo, B. Fleck, B. Battrick, Eds. (ESA SP-376, Vol. 2, ESA, Noordwijk, Netherlands, 1995), pp. 285-288] is much less expensive because it exploits the fact that the kernels are approximately just the product of a function of radius and a function of latitude (Fig. 1).

24. The RLS fit minimizes the sum of the squared residuals (the $\chi^{2}$ misfit) between the data and the splittings predicted by the parametrized model, plus a function that penalizes solutions that are large or that vary on small length scales. The competing misfit and regularity of the solution are balanced by a trade-off parameter [see I.J.D. Craig and J.C. Brown, Inverse Problems in Astronomy (Adam Hilger, Bristol, 1986)].

25. The data were splitting coefficients $a_{i}(i=1,3, \ldots, 17)$ averaged over four months, for $957(n, l)$ multiplets in the ranges $l=3-178, \nu=1500-3500 \mu \mathrm{Hz}$. Splittings were determined for each of GONG months 1, 2, 4 and 5 separately and these were then averaged with equal weights.

26. A SOLA inversion in latitude is performed for each degree $l$ to localize the angular kernels $\left[P_{l}^{m}(x)\right]^{2}$, where $P_{l}^{m}$ is an associated Legendre function and $x=\cos \theta$. The chosen target function, which is normalized to have unit area, is a sum of two gaussians, one centered at $x_{0}$ and the other at $-x_{0}$. Once the angular inversion coefficients $\beta_{l m}$ are obtained, the quantities $F_{n l}=\sum_{m} \beta_{l m} \Delta \nu_{n l m}$ are computed and stored. (For the inversion presented here splitting coefficients were used instead of the $\Delta \nu_{n l m}$, but the principle is the same.) Radial inversion coefficients $c_{n l}$ for target location $r_{0}$ are determined by minimizing an integral over $r$ and $x$ of the square of the 
averaging kernel, weighted by $\left(r-r_{0}\right)^{2}\left(x-x_{0}\right)^{2}$. Finally the rotation rate estimate is obtained as $\sum_{n l} c_{n l} F_{n l}$.

27. The implementation of the RLS inversion was as described in (21), with secondderivative smoothing in both radial and latitudinal directions. We obtain similar results using first-derivative smoothing in the latitudinal direction. The values of the trade-off parameters in the two directions were chosen to be $\mu_{r}=10^{-6}, \mu_{\theta}=10^{-5}$ so as to give a reasonable trade-off between resolution and variance of the solution. More automated procedures for choosing values for the trade-off parameters do exist, such as generalized cross validation.

28. The formal 1- $\sigma$ error bars in Fig. 4 indicate how the solution at a point would vary given different realizations of the data noise. They do not necessarily provide a confidence interval that covers the true solution, because of the finite resolution of the inversion. Specifically, in the RLS the choice of finite basis and the smoothing penalty term both introduce biases. It is possible to estimate the magnitude of the latter effect by computing the terms in an expansion about the estimated parameters, but we have not done so here.

29. T.L. Duvall, Jr., Solar Phys. 66, 213 (1980).

30. P. Foukal and J.R. Jokipp, Astrophys. J. 199, L71 (1975); P. Foukal, ibid. 218, 539 (1977); P.A. Gilman and P.V. Foukal, ibid. 229, 1179 (1979).

31. N.H. Brummell, X. Xie, J. Toomre, C. Baillie, Astron. Soc. Pacific Conf. Ser. 76, 192 (1995); J. Toomre and N.H. Brummell, in Fourth SOHO Workshop Helioseismology, T. Hoeksema, V. Domingo, B. Fleck, B. Battrick, Eds. (ESA SP-376, Vol. 1, ESA, Noordwijk, Netherlands, 1995), pp. 47-62; N.H. Brummell, N.E. Hurlburt, J. Toomre, Astrophys. J., submitted (1996).

32. Time-distance methods based on studying acoustic travel time along sets of ray paths are revealing the potential to map localized structures in wave speed and flow velocities, as discussed using HDH data from the South Pole by T.L. Duvall, Jr., S.M. Jeffries, J.W. Harvey, M.A. Pomerantz, Nature 362, 430 (1993); ——, S. D’Silva, 
S.M. Jeffries, J.W. Harvey, J. Schou, ibid. 379, 235 (1996); with inversion of maps of travel times reported by A.G. Kosovichev, Astrophys. J., in press (1996). Ringdiagram analysis involves observing the wave fields while tracking localized regions on the Sun to deduce the averaged horizontal flows and sound-speed perturbations beneath the region, as discussed by F. Hill, Astrophys. J. 333, 996 (1988); —-, Solar Phys. 128, 321 (1990); ——, Astron. Soc. Pacific Conf. Ser. 76, 484 (1995). Inversion of data measuring displacement of the rings evident in slices at fixed frequency in the power spectra are presented in J. Pátron et al., Astrophys. J. 455, 746 (1995).

33. The transport of angular momentum within the convection zone and in an adjustment layer at its base is discussed by P.A. Gilman, C.A. Morrow, E.E. DeLuca, Astrophys. J. 338, 528 (1989). The properties of a turbulent 'solar tachocline' to achieve a transition in the rotation rate of the convection zone to that of the deeper interior is discussed in E.A. Spiegel and J.-P. Zahn, Astron. Astrophys. 265, 106 (1992), assuming that the helioseismically inferred rotation profile of the convection zone must adjust rapidly in radius to the interior state while transferring little or no net torque. If shear instabilities in the adjustment layer lead to substantial turbulent diffusive processes in the horizontal that can balance the advection of angular momentum, then a thin transition region can result.

34. E.A. Spiegel and N.O. Weiss, Nature 287, 616 (1980); R. Rosner, in Cool Stars, Stellar Systems, and the Sun, A.K. Dupree, Ed. (SAO SR-389, Cambridge, 1980), pp. 79-96; L. Golub, R. Rosner, G.S. Vaiana, N.O. Weiss, Astrophys. J. 243, 309 (1981); G.A. Glatzmaier, Geophys. Astrophys. Fluid Dyn. 31, 137 (1985); D. Schmitt, in The Cosmic Dynamo, F. Krause, K.-H. Rädler, G. Rüdiger, Eds. (Kluwer, Dordrecht, Netherlands, 1993), pp. 1-12.

35. The finite resolution smooths out even discontinuous transitions [see M.J. Thompson, Solar Phys. 125, 1 (1990)]. Techniques more appropriate for detecting sharp features include looking directly for the signature of a discontinuity or jet stream [D.O. Gough in Proc. Oji Intern. Seminar Prog. of Seismology of the Sun and Stars, Y. Osaki 
and H. Shibahashi, Eds. (Springer Verlag, Berlin, 1990), pp. 283-318] and designing averaging kernels that are sensitive to sharp gradients or local extrema [F.P. Pijpers and M.J. Thompson, Astron. Soc. Pac. Conf. Ser. 42, 201 (1993)].

36. R. Rosner and N.O. Weiss, Nature 317, 790 (1985); L. Mestel and N.O. Weiss, Mon. Not. R. Astron. Soc. 226, 123 (1987); P. Charbonneau and K.B. MacGregor Astrophys. J. 417, 762 (1993).

37. N.O. Weiss, Proc. Roy. Soc. A 293, 310 (1966).

38. J.-P. Zahn, Astron. Astrophys. 288, 829 (1994).

39. E. Schatzman, Astrophys. Lett. 3, 139 (1969); ——, Astron. Astrophys. 56, 211 (1977); J.-P. Zahn, ibid. 265, 115 (1992).

40. A measure of the local resolution is the width of the main peak of the averaging kernels. We estimate the radial and latitudinal resolution by fitting to each averaging kernel a double Gaussian with widths $w_{r}$ and $w_{\theta}$ in radius and latitude, plus its mirror image in the equatorial plane (21). The widths of some representative averaging kernels for the RLS inversion illustrated in Fig. 3B and Fig. $4 \mathrm{~B}$ are: $w_{r}=0.06 R$, $w_{\theta}=0.11 R$ at $r=0.70 R, 60^{\circ} ; w_{r}=0.05 R, w_{\theta}=0.10 R$ at $r=0.70 R, 30^{\circ}$; $w_{r}=0.025 R, w_{\theta}=0.10 R$ at $r=0.91 R, 30^{\circ}$. The FWHM in each direction is larger than these values by roughly a factor of 1.7 .

41. J. Christensen-Dalsgaard, J. Schou, M.J. Thompson, J. Toomre, Astron. Soc. Pacific Conf. Ser. 76, 212 (1995); ——, in Fourth SOHO Workshop Helioseismology, T. Hoeksema, V. Domingo, B. Fleck, B. Battrick, Eds. (ESA SP-376, Vol. 2, ESA, Noordwijk, Netherlands, 1995), pp. 41-45.

42. We acknowledge financial support from the UK Particle Physics and Astronomy Research Council, the National Science Foundation, the National Aeronautics and Space Administration, Danmarks Grundforskningsfond, and the French Centre Nationnal de la Recherche Scientifique and Institut du Developpement et des Ressources en Informatique Scientifique. This work utilizes data obtained by the Global Oscillation 
Network Group (GONG) project, managed by the National Solar Observatory, a Division of the National Optical Astronomy Observatories, which is operated by AURA, Inc. under a cooperative agreement with the National Science Foundation. The data were acquired by instruments operated by the Big Bear Solar Observatory, High Altitude Observatory, Learmonth Solar Observatory, Udaipur Solar Observatory, Instituto de Astrofísica de Canarias, and Cerro Tololo Interamerican Observatory. 

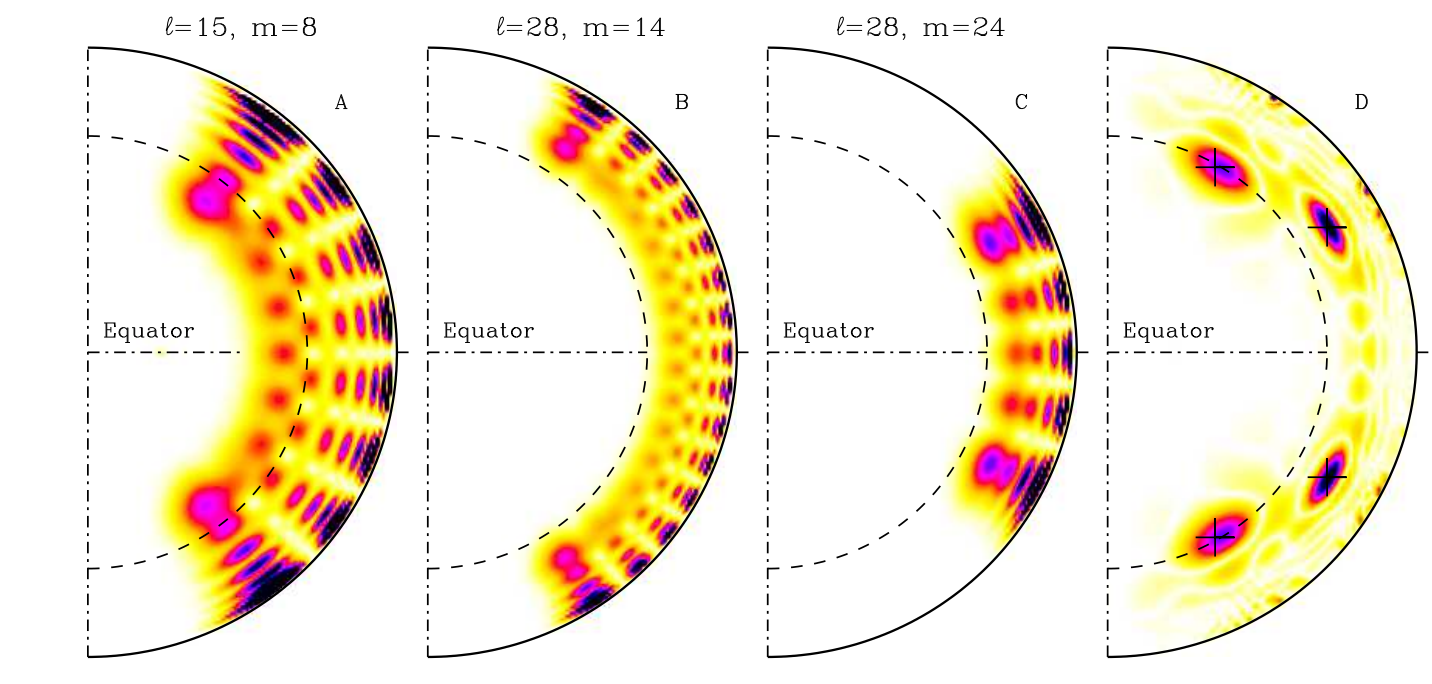

Fig. 1. Meridional cuts through three rotation kernels for modes with frequencies $\nu \approx$ $1500 \mu \mathrm{Hz}$, for (A) $l=15, m=8$, (B) $l=28, m=14$, (C) $l=28, m=24$. The latitudinal extent of the kernels varies with $m / L$, where $L=\sqrt{l(l+1)}$, and the radial extent varies with $\nu / L$. Except close to the deepest point where it has appreciable amplitude, each kernel is nearly just a product of a function of radius and a function of latitude. (D) Meridional cut through averaging kernels for a regularized least-squares inversion (as in Fig. 3B), for target radii and latitudes $r=0.70 R, 60^{\circ}$ and $r=0.82 R, 30^{\circ}$. The averaging kernels are symmetric about the equatorial plane, so that they are also peaked at latitudes $-60^{\circ}$ and $-30^{\circ}$. 


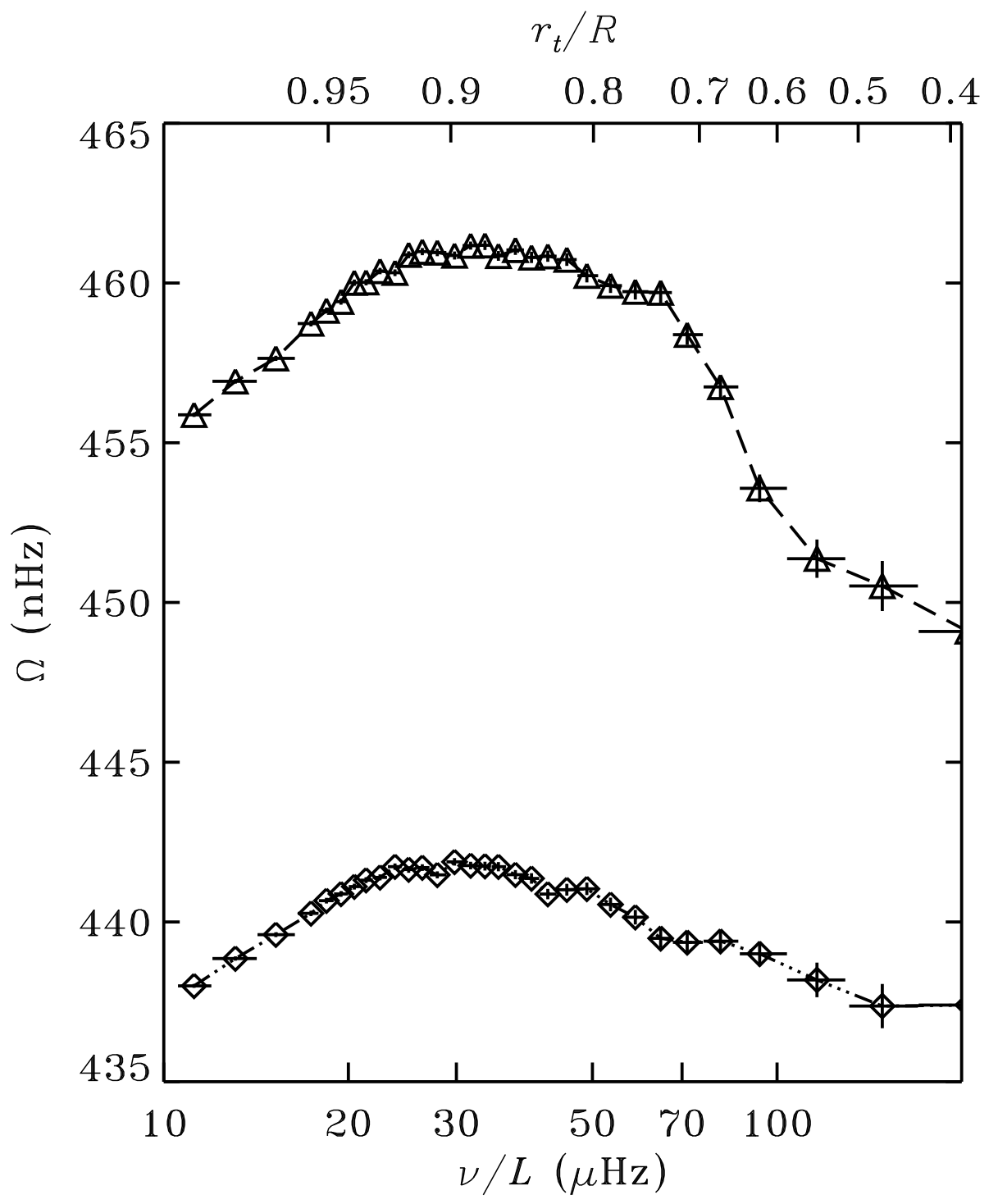

Fig. 2. Combinations of the first three odd splitting coefficients (20) corresponding to the equatorial region (upper curve) and latitude $30^{\circ}$ (lower), plotted as functions of $\nu / L$, where $\nu$ is frequency and $L=\sqrt{l(l+1)}$. The values are roughly a weighted average of the rotation at those latitudes, between the surface and the lower turning point radius $r_{t}$ (top axis). The average rotation in the equatorial region is greater than at latitude $30^{\circ}$, and also at both latitudes the rotation initially increases with depth below the surface, before decreasing at greater depth. 

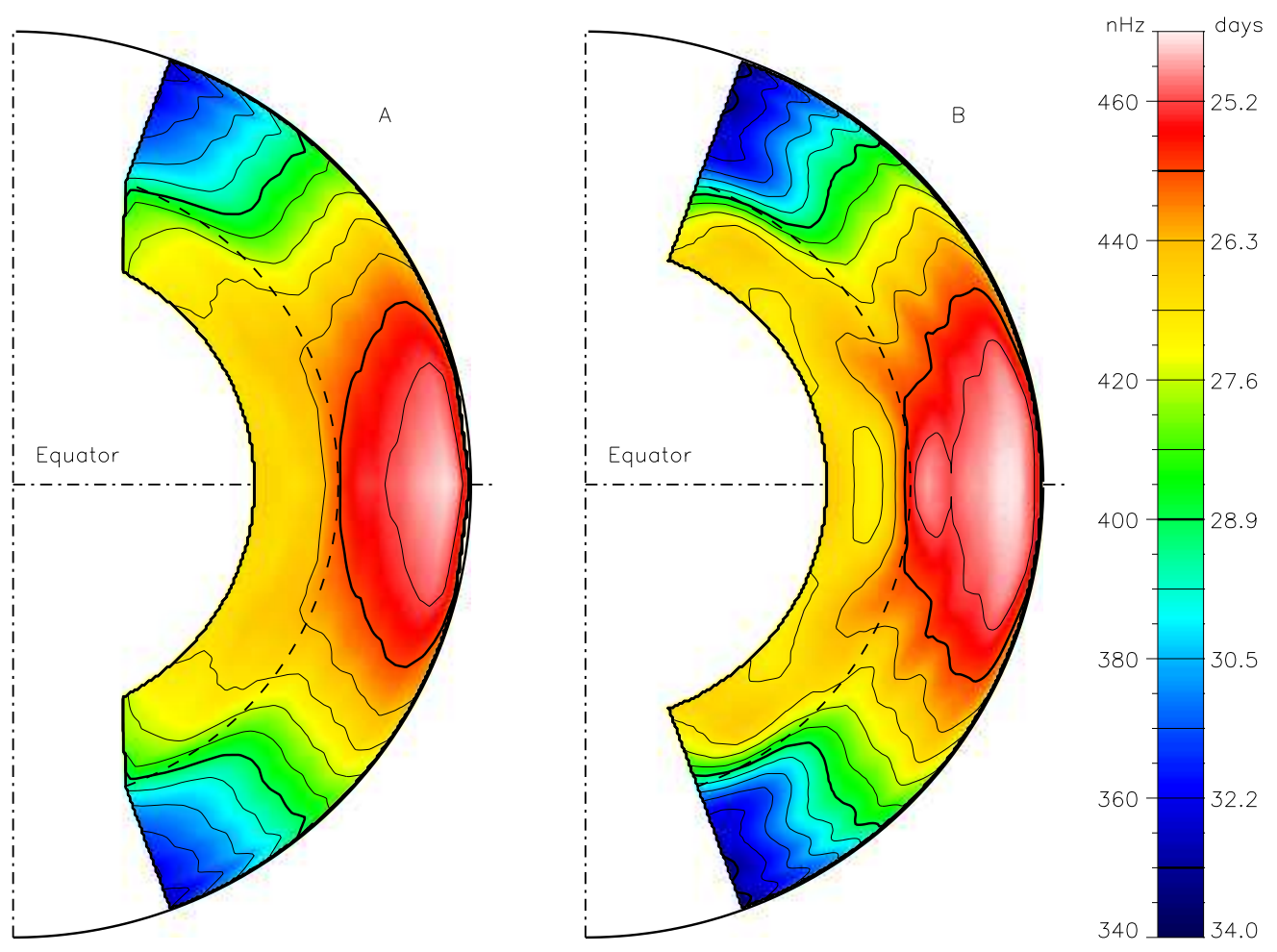

Fig. 3. The inferred sidereal rotation rate inside the Sun from 4 months of GONG data, based on (A) an OLA $1 \otimes 1$ inversion (23, 26), and (B) an RLS inversion (27). Lighter shading corresponds to faster rotation; the contour spacing is $10 \mathrm{nHz}$, the highest contour value being $460 \mathrm{nHz}$. [Need to revise to discuss instead the meaning of colors.] The approximate base of the convection zone is indicated by the dashed line at $r=0.7 R$. The slightly more jagged appearance of the RLS solution results from a different balance between resolution and noise in the two inversions. Particularly noticeable is the local maximum in the rotation rate a little below the surface in the equatorial region. This shear layer appears to persist at least to mid-latitudes. 

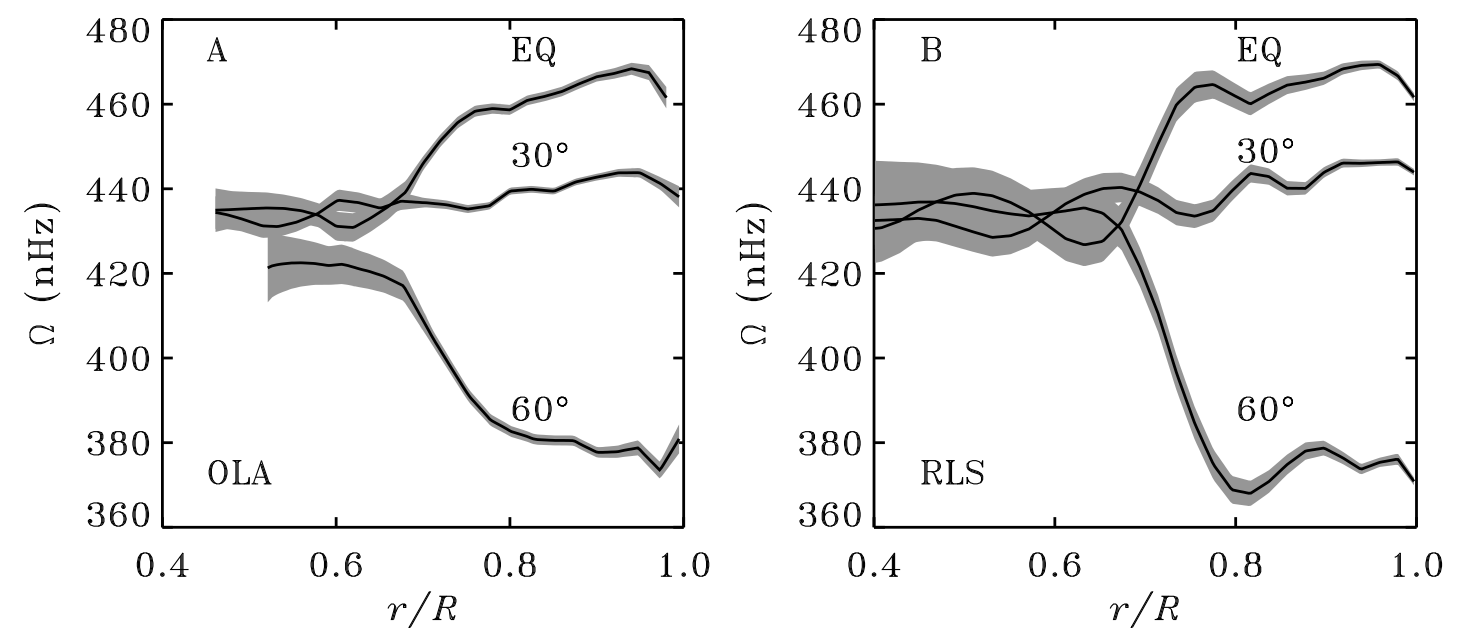

Fig. 4. Sections at latitudes $0^{\circ}$ (solid), $30^{\circ}$ (dashed) and $60^{\circ}$ (dotted) through (A) the OLA and (B) the RLS inversion solutions shown in Fig. 3. Also indicated are formal \pm 1 standard deviation error bars (28). Both inversions indicate that surface-like differential rotation persists through the bulk of the convection zone, with a transition near the base of the convection zone to a flow that is consistent with latitudinally-independent rotation. 

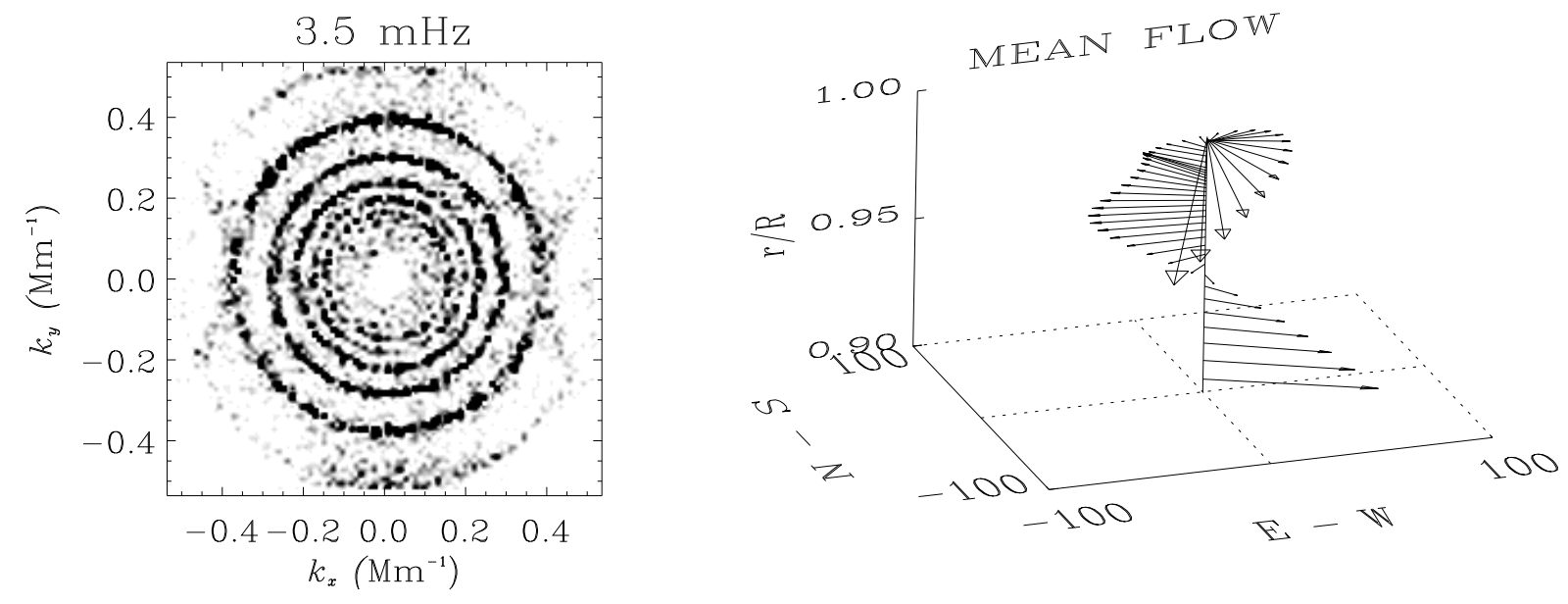

Fig. 5. Ring-diagram analysis of the superposition of local acoustic plane waves, each of which is advected locally by the horizontal component of the mean flow beneath the solar surface. The procedure here uses GONG multi-station data in which a $45^{\circ}$ subraster (involving $96^{2}$ pixels and centered on the equator) is tracked at the solar rotation rate over a 56 hour interval. (A) Multiple rings (corresponding to different values of order $n$ ) are evident in the cut through $k_{x}-k_{y}-\nu$ power spectrum at constant frequency $\nu=3.5 \mathrm{mHz}$, where $k_{x}$ and $k_{y}$ are the eastward and northward components of the horizontal wavenumber. (B) The displacement of such rings can be used to infer the effective mean horizontal velocity sensed by the acoustic waves over a range of depths (32), shown as the spiralling set of arrows denoting amplitude and direction of that mean flow (in $\mathrm{m} \mathrm{s}^{-1}$ ) with proportional radius $r / R$ just below the surface. 